\title{
Can Changes in Skin Impedance Be Used to Monitor Sedation After Midazolam and During Recovery From Anesthesia?
}

\author{
Alice KURZOVÁ ${ }^{1}$, Ladislav HESS ${ }^{2}$, Jiří SLÍVA ${ }^{3}$, Jiří MÁLEK ${ }^{1}$ \\ ${ }^{1}$ Department of Anesthesiology and Critical Care Medicine, Third Faculty of Medicine, Charles \\ University in Prague, and University Hospital Kralovske Vinohrady, Prague, Czech Republic, \\ ${ }^{2}$ Institute for Clinical and Experimental Medicine, Laboratory of Experimental Anesthesiology, \\ Prague, Czech Republic, ${ }^{3}$ Department of Pharmacology, Third Faculty of Medicine, Charles \\ University in Prague, Czech Republic
}

Received November12, 2020

Accepted January 4, 2021

Epub Ahead of Print March 8, 2021

\section{Summary}

It has been suggested that sympathetic activity, measured as changes in electrical skin impedance (SI), can be used to assess the adequacy of general anesthesia. Our prospective study investigated if measurements of skin impedance can determine levels of sedation induced by midazolam. Twenty-seven patients scheduled for arthroscopy requiring general anesthesia were served as their own control. These were blinded to the order of injections by telling them that they will be randomly administered a placebo (saline) or sedative agent. A DM 3900 multimeter was used for SI measurements. The degree of sedation was measured using the modified Observer's Assessment of Alertness and Sedation (mOAAS) scale. Resting SI values were noted, and all participants were then administered the placebo followed $5 \mathrm{~min}$ later by midazolam $2 \mathrm{mg}$ i.v. Five min after that, patients were administered standard general anesthesia with propofol, oxygen, nitrous oxide $60 \%$, and isoflurane 1 MAC via a laryngeal mask, and sufentanil 5 - $10 \mu \mathrm{g}$. SI significantly increased after administration of midazolam and induction of anesthesia. There were no significant differences between pre-administration (baseline) and placebo and end of surgery and end of anesthesia with closed eyes. There were highly significant differences $(p<0.001)$ between pre-administration vs. midazolam, placebo vs. midazolam, pre-administration vs. induction of anesthesia. We found slight correlation between mOAAS and SI. There were no significant changes between the end of surgery and the end of anesthesia with closed eyes, but SI significantly decreased $(p<0.01)$ after eyes opened.

\section{Key words}

Anesthesia • Midazolam • Skin impedance • Sedation

\section{Corresponding author}

Jiri Sliva, Dpt. of Pharmacology, Charles University, Ruská 87, 10000 Prague, Czech Republic. E-mail: Jiri.Sliva@lf3.cuni.cz

\section{Introduction}

Sedation is frequently used for a variety of procedures performed outside the operating rooms by non-anesthetists. Usually, anxiolysis is preferable to sedation since sedation may interfere with recovery after short procedures and may bring the risk of inadvertent deep sedation with loss of airway patency. Unfortunately, although anxiolytic sedatives are widely used in clinical practice, the methodology for assessing the treatment effect of these compounds has not been well developed. Psychological tests like the Hamilton Anxiety Rating Scale (HARS), the Wang Anxiety Rating Scale (WARS), or the Zung Self-Rating Anxiety Scale (SAS) have been used to assess changes in anxiety, but they were developed to measure the effect of long-term therapy and are rather cumbersome and difficult to use in routine clinical practice.

Tests such as the Ramsay sedation scale (RSS), the Richmond Agitation-Sedation Scale (RASS), and the Observer's Assessment of Alertness and Sedation (OAAS) scale are commonly used in perioperative 
clinical settings, but they focus mainly on the level of sedation and not the level of anxiolysis. There are few methods available for assessing the onset and intensity of sedation. A bispectral index (BIS) monitor is commonly used as an objective measure of the "alert" state, although mainly during general anesthesia. A large meta-analysis concluded that its use for sedation was largely unreliable (Shetty et al. 2018, Zhang et al. 2019).

There have been several studies demonstrating a correlation between the level of anxiety and changes in skin impedance. Changes of skin impedance were first used in criminology in 1897. In 1921 August Larson combined changes in SI with changes in heart rate, respiratory rate, and blood pressure to create a device for use by law enforcement (Marsh 2019, Widacki 2015). This method has been validated many times, and these devices, polygraphs, are still used as "lie detectors." More recently, several studies have demonstrated a correlation between changes in skin impedance and the degree of sedation, the bispectral index, reaction to stimulation, pain, and motor activity (Cowen et al. 2015, Gjerstad et al. 2007, Winterhalter et al. 2008, 2009, 2010). A change in skin impedance is a complex response to sympathetic nervous system (SNS) activity and depends on emotional states like fear, anxiety, and pain. SNS activity can be measured using secretions from eccrine sweat glands. Various drugs like sedatives, opioids, and anesthetics act through central mechanisms that depress
SNS activity, which is then followed by changes in skin impedance (Gjerstad et al. 2007, Winterhalter et al. 2010).

The present double-blind study was designed to refine the methodology for evaluating the effect of sedative and anxiolytic agents by assessing drug-related SNS-mediated changes in SI.

The primary aim of our study was to determine if skin impedance can be used as an objective measurement of the level of sedation compared to the, more or less, subjective OAAS. The OAAS was chosen because the scoring system was initially introduced to assess sedation with benzodiazepines, which were used (midazolam) in our study. The OAAS categories were responsiveness, speech, facial expression and eye appearance, and each of the categories was scored on 5 dimensions. The OAAS scale was found to have high discriminatory power for the different levels of sedation (Chernik et al. 1990), but may vary between assessors. Modified OAAS (mOAAS) (Nonaka et al. 2018) was used in our study because of better reflection of changes during deep level of sedation and anesthesia (Table 1). Midazolam was used in our trial because it is the most commonly used drug to reduce anxiety before anesthesia and surgery.

A secondary study goal was to investigate if skin impedance can help to determine the level of onset and recovery from general anesthesia.

Table 1. Modified Observer's Assessment of Alertness/Sedation Scale (mOAAS). Available via license: CC BY-NC-ND

\begin{tabular}{cl}
\hline Scores & \multicolumn{1}{c}{ Description } \\
\hline 5 & Fully awake. Responds readily to name spoken in normal tone \\
4 & Drowsy. Lethargic responds to name spoken in normal tone \\
3 & Eyes closed. Responds only after name is called loudly and/or repeatedly \\
2 & Responds only after mild prodding or shaking \\
1 & Does not respond to mild prodding or shaking \\
0 & Does not respond to deep stimulus \\
\hline
\end{tabular}

\section{Methods}

The study was approved by the Ethics Committee of the University Hospital Kralovske Vinohrady and is registered at ClinicalTrials.gov as NCT03791424.

\section{Subjects}

The study was performed on adult patients scheduled for knee arthroscopy under general anesthesia.
Consent was obtained the day before surgery during the pre-anesthetic visit. Exclusion criteria were a previous history of taking drugs affecting mental functions, history of mental illness, an implanted electrical device, allergy to benzodiazepines, myasthenia gravis, an American Society of Anesthesiologists (ASA) classification of ASA 3 or higher. All patients consented to not use any medication for sedation or anxiety, opioid analgesics, ethanol, and/or illicit drugs within 24 hours before 
surgery. Patients were instructed that they would be administered, in random order, a sedative agent or placebo, and would be observed for their level of sedation.

\section{Measurements}

After arrival at the operating theater and venipuncture, two standard $\mathrm{AgCl}$ ECG electrodes (Medico Electrodes International ltd.) size $27 \mathrm{~mm}$ x 35 $\mathrm{mm}$ were attached to the palm side of the second and third finger of the non-dominant hand. A DM 3900 Multimeter (Mastech) was used to measure SI. The device was controlled and approved for use on humans by the Department of Medical Biophysics and Informatics of the Third Faculty of Medicine of Charles University.

As described above, the modified OAAS (mOAAS) scale was used as a standard tool to measure sedation levels. It was administered at the same times as the SI measurements were taken. The person providing the mOAAS assessment was also blinded to the order of midazolam and placebo injection, and only the anesthesiologist administering the injections knew the order of drugs.

Data recording: Participants were given $10 \mathrm{~min}$ to acclimate to the test situation. The resting SI value for each patient was noted. Despite the instructions to the contrary, all patients were administered the placebo first, followed $5 \mathrm{~min}$ later by midazolam $2 \mathrm{mg}$ i.v., so they could serve as their own controls. After another $5 \mathrm{~min}$, patients were administered standard general anesthesia with propofol, oxygen, nitrous oxide $60 \%$, and isoflurane 1 MAC (adjusted to the age and BMI of the patient) via a laryngeal mask, and sufentanil $5-10 \mu \mathrm{g}$ as needed. Monitoring was routine for this type of surgery (i.e., ECG, non-invasive blood pressure, capnography, and pulse oximetry). SI and OAAS were recorded at the end of the surgery (i.e., after stopping the volatile anaesthetic agent), then with zero exhaled isoflurane (i.e., the end of anesthesia with eyes closed) and the end of anesthesia with the patient's eyes open and verbally engaged.

\section{Statistical analysis}

SI values before placebo administration (baseline), $5 \mathrm{~min}$ after placebo, $5 \mathrm{~min}$ after midazolam, 5 min after induction, at the end of the surgery, zero exhaled isoflurane after anesthesia with closed eyes, and after eyes opened were recorded. Changes in SI values compared to baseline data were used for analyses using a one-way ANOVA with the post-hoc Tukey's test. These relative changes allowed us to compare the course of changes independently of the absolute values. Pearson's correlation coefficients were used to analyze the relationship between mOAAS and SI values. P-values $<0.05$ were considered statistically significant.

\section{Results}

All 30 patients eligible for the study consented, but because of two postponed surgeries, only 28 patients were analyzed. There were 14 men and 14 women. The mean age was 39.8 years (standard deviation (SD) was $9.3 \mathrm{y}$ ), the mean BMI was $26.2 \mathrm{~kg} / \mathrm{m}^{2}\left(\mathrm{SD}=4.4 \mathrm{~kg} / \mathrm{m}^{2}\right)$, and the mean duration of surgery was $25.5 \mathrm{~min}$. $(\mathrm{SD}=16.5 \mathrm{~min})$.

\section{Primary outcome measures}

SI significantly increased after administration of midazolam and induction of anesthesia, although the inter-individual baseline skin impedance values differed greatly from patient to patient. For this reason, percent changes from baseline were used instead of absolute values. There were no significant differences between pre-administration vs. placebo and end of surgery vs. end of anesthesia with closed eyes. Highly significant differences were seen between the first two and the last measured points (i.e., $5 \mathrm{~min}$ after placebo and 5 min after midazolam, and end after anesthesia with eyes open) and the fourth and fifth measured points (i.e., at the end of surgery vs. zero exhaled isoflurane, end of anesthesia with closed eyes) $-\mathrm{p}<0.001$ for all comparisons. Additional significant differences involved comparison between measurements at $5 \mathrm{~min}$ after induction and $5 \mathrm{~min}$ after placebo $(p=0.02)$, and at the end of surgery $(p=0.003)$ and at zero exhaled isoflurane, end of anesthesia with eyes closed ( $p=0.01)$ (Fig. 1). There was a slight correlation between the mOAAS and SI. The mOAAS decreased from approximately 5.0 before the administration of midazolam to 4.2 after midazolam administration. Correlations between relative skin impedance and mOAAS are shown in Fig. 2. There was some gender difference in the relative changes in skin impedance. Except of $\mathrm{CE}(\mathrm{p}<0.05)$, all of them were statistically non-significant (Fig. 3).

\section{Secondary outcome measures}

There were no significant changes between the end of surgery and the end of anesthesia with eyes closed, but SI significantly decreased $(p<0.01)$ after eyes opened. 



Fig. 1. Relative changes in skin impedance from base line (i.e., prior to placebo administration). Legend: placebo - $5 \mathrm{~min}$ after placebo, MDZ - 5 min after midazolam, GA - 5 min after induction, EOS - at the end of surgery, CE - zero exhaled isoflurane, end of anesthesia with eyes closed, OE - end of anesthesia, eyes open. Bars represent means with whiskers showing \pm standard errors of means ${ }^{* * *} \mathrm{p}<0.001$ vs. placebo, MDZ, and $\mathrm{OE}^{* *} \mathrm{p}=0.003$ vs. EOS; ${ }^{++} \mathrm{p}=0.01$ vs. CE; ${ }^{++} \mathrm{p}=0.02$ vs. placebo)

Fig. 2. Correlations between relative skin impedance and mOAAS. Bars represent means with whiskers showing \pm standard errors of means
Fig. 3. Gender differences relative to changes in skin impedance. Bars represent means with whiskers showing \pm standard errors of means $\left({ }^{+} p=0.05\right)$

\section{Discussion}

Assessments of the depth of sedation are usually dependent on verbal or motor responses to various stimulations and are dependent on the subjective skills of the assessor. The use of stimuli can potentially result in patient responses that disrupt the procedure for which the patient is sedated. Various objective methods have been tried to assess the level of anxiolysis and light sedation.
The goal is to find an objective measure of the level of consciousness, which, if valid, would improve the quality of conscious sedation (Chisholm et al. 2006). Ideal monitoring of sedation levels would have several potential advantages over observational clinical sedation scoring systems: it would be objective, quantitative, easy to use, free of observer bias, economical, and noninvasive. Objective monitoring usually uses EEG-based measurements, which were developed mainly to avoid 
alertness during general anesthesia. There are some works comparing subjective and objective methods like the bispectral index (BIS) monitor or the Patient State Analyzer; however, for the area of clinical interest, i.e., mild sedation, they are not sufficiently sensitive (Chisholm et al. 2006). Very similar results were obtained by Haberland et al. (2011), who used BIS to assess levels of sedation in children (Haberland et al. 2011). Correlations between BIS and clinical observations were statistically significant, but only moderately so. There were several children observed to be in deep sedation while the BIS monitor showed alertness. Results of the Cochrane review (Shetty et al. 2018) also found that BIS measurements had little value relative to ICU sedated patents. Recently, two monitors for measurement pain, depth of analgesia and anesthesia using relative changes of SI as a part of global calculated scale e.g. NOL index (PMD-200TM, Medasense, Ramat Gan, Israel) and NFSC index (Skin Conductance Algesimeter, MedStorm innovations, Oslo, Norway) has introduced to clinical practice, but they are intended for monitoring of reaction to pain stimulation during surgery (Rogoyov and Vanek 2020).

Our results showed that skin impedance could be used to measure levels of anxiety and sedation. Changes in skin impedance can provide complex information regarding anxiety or fear and depressed levels of consciousness.

Sympathetic activation by cortical and subcortical areas in the brain produce changes in sweat gland activity, which are exclusively innervated by the SNS (Gjerstad 2012). There is a direct correlation between eccrine sweat gland activity and skin impedance (Winterhalter et al. 2008). There is also a direct correlation between the discharge rate during sympathetic nerve stimulation and the amplitude of simultaneously measured skin impedance changes (Bach 2014). There have been several attempts to use skin impedance to assess specific parts of this complex state, but the results were inconsistent. Measurements of skin impedance or conductance have also been used to assess pain intensity or the quality of analgesia. Storm et al. recommended using skin conductance, instead of heart rate, blood pressure, and electroencephalographs (EEG), as a more sensitive tool for measuring pain perception since it is independent of anesthesia level and muscle relaxation (Storm 2008). Czaplik et al. tried using changes in skin conductance as a way to target acute pain therapy in the post-anesthesia care unit; however, they failed to find any long-term correlation between the numeric pain rating scale and the number of fluctuation in skin conductance (NFSC) (Czaplik et al. 2012). They concluded that there are many other factors affecting SI results, e.g., anxiety, level of sedation, disorientation, shivering, etc., and that this method works best for sedated patients. Similar results were seen in other studies. Ledowski et al. demonstrated a high correlation between NFSC and pain ratings, although with several limitations, mainly the fact that many drugs used for sedation and anesthesia may influence the result (Ledowski et al. 2007). In a later study from 2010, Ledowski et al. failed to find any correlation between stress hormones levels and skin conductance (Ledowski et al. 2010). More recently, Valkenburg et al. (2012) drew similar conclusions with infants, and Günther et al. found the same with ICU adult patients (Gunther et al. 2013). The latter concluded that NFSC might be more influenced by anxiety and stress than by pain intensity. These findings support our opinion that factors responsible for skin impedance changes are complex, and SI is probably best suited for objective assessment of anxiety and sedation level. The interpretation of the differences between men and women (Fig. 3) is difficult. We can only speculate that it is a result of different sympathetic nerve regulation (Hinojosa-Laborde et al. 1999, Kittnar 2020, Ueno et al. 2003). Gender differences were described also in pain perception and the reasons are probably multifactorial (Rokyta and Yamamotova 2013).

Several studies measured skin impedance during anesthesia and compared it to ECG methods. Winterhalter et al. demonstrated that skin impedance correlated to the depth of sedation during targetedcontrolled infusion of propofol and was correlated with both BIS and mOAAS values in 22 patients and 8 healthy volunteers. In a second study, Winterhalter et al. measured skin impedance changes during anesthesia induction with fentanyl and etomidate and compared the results with BIS values. In spite of using different drugs and a slightly different protocol (i.e., concentrating mainly on the induction of anesthesia), their results correspond with our findings (Winterhalter et al. 2009, Winterhalter et al. 2010).

We did not confirm the usefulness of skin impedance measurements for assessing depth and emergence from aesthesia; however, Gjerstad et al. (Gjerstad et al. 2007) found a correlation between NFSC and Response Entropy (RE), which measures electromyographic and electroencephalographic activity 
from electrodes placed on the forehead. However, in this study, changes in skin impedance during emergence from anesthesia were slower than RE changes. In one of their 20 patients, the NFSC was unchanged $1 \mathrm{~min}$ after extubation.

\section{Limitations}

Our study has several limitations. The first is a study design in which the same patients were used for both placebo and active treatment. However, the variability in absolute values of skin impedance made it the only method suitable for testing the difference between the placebo and active treatment. The second limitation involved the interpretation of values. A high level of inter-individual variability prevents the use of absolute values, and the percentage change over time had to be used. In the context of the comparability of skin impedance measurements, relative changes from an individual patient's baseline value had to be used. Another issue, which was mentioned by several other authors, involved minor differences linked to the electrodes, e.g., changes in hydration under electrodes, an absence of sweat gland activity, and sweat accumulation under electrodes during heavy perspiration. For the purpose of our study, the administration of atropine and other drugs used during the maintenance of anesthesia and emergence from anesthesia with theoretical exception of small dose of sufentanil in all patients were not relevant to the outcomes, as they were not used.

\section{Conclusions}

Changes of skin impedance can be used as a simple and economical method to measure the onset of anxiolysis and sedation induced by midazolam; additionally, SI correlated with mOAAS scores. However, until this use of SI will be more and deeply studied, we cannot recommend it for the assessment of the depth of anesthesia and for predicting emergence from anesthesia.

\section{Conflict of Interest}

There is no conflict of interest.

\section{Acknowledgements}

The authors wish to acknowledge Prof. Jozef Rosina, MD, Ph.D., MBA from the Department of Medical Biophysics and Informatics of the Third Medical Faculty of Charles University for his technical help. This work was supported by PROGRESS Q 35.

\section{References}

BACH DR: Sympathetic nerve activity can be estimated from skin conductance responses - a comment on Henderson et al. (2012). Neuroimage. 84: 122-123, 2014. https://doi.org/10.1016/j.neuroimage.2013.08.030

CHERNIK DA, GILLINGS D, LAINE H, HENDLER J, SILVER JM, DAVIDSON AB, SCHWAM EM, SIEGEL JL: Validity and reliability of the Observer's Assessment of Alertness/Sedation Scale: study with intravenous midazolam. J Clin Psychopharmacol 10: 244-251, 1990. https://doi.org/10.1097/00004714-199008000-00003

CHISHOLM CJ, ZURICA J, MIRONOV D, SCIACCA RR, ORNSTEIN E, HEYER EJ: Comparison of electrophysiologic monitors with clinical assessment of level of sedation. Mayo Clin Proc 81: 46-52, 2006. https://doi.org/10.4065/81.1.46

COWEN R, STASIOWSKA MK, LAYCOCK H, BANTEL C: Assessing pain objectively: the use of physiological markers. Anaesthesia 70: 828-847, 2015. https://doi.org/10.1111/anae.13018

CZAPLIK M, HUBNER C, KONY M, KALICIAK J, KEZZE F, LEONHARDT S, ROSSAINT R: Acute pain therapy in postanesthesia care unit directed by skin conductance: a randomized controlled trial. PLoS One 7: e41758-2012. https://doi.org/10.1371/journal.pone.0041758

GJERSTAD AC, STORM H, HAGEN R, HUIKU M, QVIGSTAD E, RAEDER J: Comparison of skin conductance with entropy during intubation, tetanic stimulation and emergence from general anaesthesia. Acta Anaesthesiol Scand 51: 8-15, 2007. https://doi.org/10.1111/j.1399-6576.2006.01189.x

GJERSTAD AC: Is skin conductance a predictor of arousal, noxious stimuli and pain in the sedated and anaesthetized patient? Series of dissertations submitted to the Faculty of Medicine, University of Oslo n. 1249, ISBN 978-828264-177-72012. 
GUNTHER AC, BOTTAI M, SCHANDL AR, STORM H, ROSSI P, SACKEY PV: Palmar skin conductance variability and the relation to stimulation, pain and the motor activity assessment scale in intensive care unit patients. Crit Care 17: R51-2013. https://doi.org/10.1186/cc12571

HABERLAND CM, BAKER S, LIU H: Bispectral index monitoring of sedation depth in pediatric dental patients. Anesth Prog 58: 66-72, 2011. https://doi.org/10.2344/0003-3006-58.2.66

HINOJOSA-LABORDE C, CHAPA I, LANGE D, HAYWOOD JR: Gender differences in sympathetic nervous system regulation. Clin Exp Pharmacol Physiol 26: 122-126, 1999. https://doi.org/10.1046/j.1440-1681.1999.02995.x

KITTNAR O: Selected sex related differences in pathophysiology of cardiovascular system. Physiol Res 69: 21-31, 2020. https://doi.org/10.33549/physiolres.934068

LEDOWSKI T, BROMILOW J, WU J, PAECH MJ, STORM H, SCHUG SA: The assessment of postoperative pain by monitoring skin conductance: results of a prospective study. Anaesthesia 62: 989-993, 2007. https://doi.org/10.1111/j.1365-2044.2007.05191.x

LEDOWSKI T, PASCOE E, ANG B, SCHMARBECK T, CLARKE MW, FULLER C, KAPOOR V: Monitoring of intra-operative nociception: skin conductance and surgical stress index versus stress hormone plasma levels. Anaesthesia 65: 1001-1006, 2010. https://doi.org/10.1111/j.1365-2044.2010.06480.x

MARSH A: A Brief History of the Lie Detector. https://spectrum.ieee.org/tech-history/heroic-failures/a-brief-historyof-the-lie-detector.Accessed 31/10/2020.2019.

NONAKA T, INAMORI M, MIYASHITA T, INOH Y, KANOSHIMA K, HIGURASHI T, OHKUBO H, IIDA H, FUJITA K, KUSAKABE A, GOTOH T, NAKAJIMA A: Can sedation using a combination of propofol and dexmedetomidine enhance the satisfaction of the endoscopist in endoscopic submucosal dissection? Endosc Int Open 6: E3-E10, 2018. https://doi.org/10.1055/s-0043-122228

ROGOYOV V, VANEK T: The importance and options of perioperative evaluation of nociception (in Czech). Anest Intenziv Med 31: 225-232, 2020.

ROKYTA R, YAMAMOTOVA A: Sex differences in pain perception and interpretation. Act Nerv SuperRediviva 55: 125-134, 2013.

SHETTY RM, BELLINI A, WIJAYATILAKE DS, HAMILTON MA, JAIN R, KARANTH S, NAMACHIVAYAM A: BIS monitoring versus clinical assessment for sedation in mechanically ventilated adults in the intensive care unit and its impact on clinical outcomes and resource utilization. Cochrane Database Syst Rev 2: CD011240-2018.

STORM H: Changes in skin conductance as a tool to monitor nociceptive stimulation and pain. Curr Opin Anaesthesiol 21: 796-804, 2008. https://doi.org/10.1097/ACO.0b013e3283183fe4

UENO T, TREMBLAY J, KUNES J, ZICHA J, DOBESOVA Z, PAUSOVA Z, DENG AY, SUN Y, JACOB HJ, HAMET P: Gender-specific genetic determinants of blood pressure and organ weight: pharmacogenetic approach. Physiol Res 52: 689-700, 2003.

VALKENBURG AJ, NIEHOF SP, VAN DM, VERHAAR EJ, TIBBOEL D: Skin conductance peaks could result from changes in vital parameters unrelated to pain. Pediatr Res 71: 375-379, 2012. https://doi.org/10.1038/pr.2011.72

WIDACKI J: Discoverers of the Galvanic Skin Response. Eur Polygraph 9: 209-220, 2015. https://doi.org/10.1515/ep2015-0008

WINTERHALTER M, MUNTE S, GERHARD M, DANZEISEN O, JUTTNER T, MONACA E, HOY L, RAHEMEYER N, KIENBAUM P: Prospective study comparing skin impedance with EEG parameters during the induction of anaesthesia with fentanyl and etomidate. Eur J Med Res 15: 47-53, 2010. https://doi.org/10.1186/2047-783X-15-2-47

WINTERHALTER M, MUNTE S, TASCHENBRECKER P, HECKER H, WEILBACH C, OSTHAUS AW, GROSS M, PIEPENBROCK S, RAHE-MEYER N: Skin impedance correlates to sedation grade, plasma propofol concentrations and bispectral index during a target-controlled infusion of propofol. Eur J Anaesthesiol 26: 589-596, 2009. https://doi.org/10.1097/EJA.0b013e328329b149

WINTERHALTER M, SCHILLER J, MUNTE S, BUND M, HOY L, WEILBACH C, PIEPENBROCK S, RAHEMEYER N: Prospective investigation into the influence of various stressors on skin impedance. J Clin Monit Comput 22: 67-74, 2008. https://doi.org/10.1007/s10877-007-9107-7 
ZHANG H, LU Y, WANG L, LV J, MA Y, WANG W, LI G, LI Y: Bispectral index monitoring of sedation depth during endoscopy: a meta-analysis with trial sequential analysis of randomized controlled trials. Minerva Anestesiol 85: 412-432, 2019. https://doi.org/10.23736/S0375-9393.18.13227-5 